\title{
Correction to: FungalTraits: a user friendly traits database of fungi and fungus-like stramenopiles
}

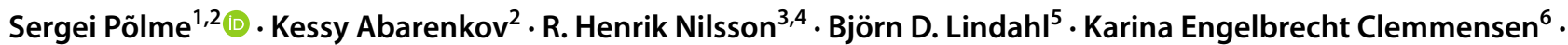
Havard Kauserud $^{7}$. Nhu Nguyen ${ }^{8} \cdot$ Rasmus Kjøller $^{9} \cdot$ Scott T. Bates $^{10} \cdot$ Petr Baldrian ${ }^{11} \cdot$ Tobias Guldberg Frøslev $^{12}$. Kristjan Adojaan $^{1}$ - Alfredo Vizzini ${ }^{13} \cdot$ Ave Suija $^{1}$. Donald Pfister ${ }^{14} \cdot$ Hans-Otto Baral $^{15} \cdot$ Helle Järv $^{16}$. Hugo Madrid ${ }^{17,18}$. Jenni Nordén ${ }^{19}$. Jian-Kui Liu ${ }^{20}$ • Julia Pawlowska ${ }^{21} \cdot$ Kadri Põldmaa $^{1} \cdot$ Kadri Pärtel $^{1}$. Kadri Runnel $^{1} \cdot$ Karen Hansen $^{22} \cdot$ Karl-Henrik Larsson $^{3,23} \cdot$ Kevin David Hyde $^{24} \cdot$ Marcelo Sandoval-Denis $^{25}$. Matthew E. Smith ${ }^{26}$ - Merje Toome-Heller ${ }^{27}$. Nalin N. Wijayawardene ${ }^{28} \cdot$ Nelson Menolli Jr. ${ }^{29,30}$. Nicole K. Reynolds ${ }^{26}$ - Rein Drenkhan ${ }^{31}$. Sajeewa S. N. Maharachchikumbura ${ }^{20}$ - Tatiana B. Gibertoni ${ }^{32}$. Thomas Læssøe ${ }^{33}$. William Davis ${ }^{34} \cdot$ Yuri Tokarev $^{35}$. Adriana Corrales ${ }^{36}$. Adriene Mayra Soares ${ }^{37} \cdot$ Ahto Agan $^{1}$. Alexandre Reis Machado ${ }^{32}$. Andrés Argüelles-Moyao ${ }^{38}$. Andrew Detheridge ${ }^{39} \cdot$ Angelina de Meiras-Ottoni $^{32}$. Annemieke Verbeken ${ }^{40}$. Arun Kumar Dutta ${ }^{41}$. Bao-Kai Cui ${ }^{42}$. C. K. Pradeep ${ }^{43}$. César Marín ${ }^{44,45}$. Daniel Stanton ${ }^{46}$. Daniyal Gohar ${ }^{1}$. Dhanushka N. Wanasinghe ${ }^{47}$. Eveli Otsing ${ }^{1}$. Farzad Aslani ${ }^{1}$. Gareth W. Griffith ${ }^{39}$.

Thorsten H. Lumbsch ${ }^{48}$ • Hans-Peter Grossart ${ }^{49,50}$. Hossein Masigol ${ }^{51} \cdot$ Ina Timling ${ }^{52}$. Inga Hiiesalu ${ }^{1}$. Jane Oja ${ }^{1}$. John Y. Kupagme ${ }^{1}$. József Geml ${ }^{53}$ • Julieta Alvarez-Manjarrez ${ }^{38} \cdot$ Kai Ilves $^{1} \cdot$ Kaire Loit $^{54} \cdot$ Kalev Adamson $^{31}$.

Kazuhide $\mathrm{Nara}^{55} \cdot$ Kati Küngas $^{1} \cdot$ Keilor Rojas-Jimenez $^{56} \cdot$ Krišs Bitenieks $^{57} \cdot$ László Irinyi $^{58,59} \cdot$ László G. Nagy $^{60}$. Liina Soonvald $^{54} \cdot$ Li-Wei Zhou $^{61} \cdot$ Lysett Wagner $^{62} \cdot$ M. Catherine Aime $^{63} \cdot$ Maarja Öpik $^{1}$ - María Isabel Mujica ${ }^{64}$. Martin Metsoja ${ }^{1} \cdot$ Martin Ryberg $^{65} \cdot$ Martti Vasar $^{1} \cdot$ Masao Murata $^{55} \cdot$ Matthew P. Nelsen $^{48} \cdot$ Michelle Cleary $^{66}$. Milan C. Samarakoon ${ }^{24} \cdot$ Mingkwan Doilom $^{47} \cdot$ Mohammad Bahram $^{1,67} \cdot$ Niloufar Hagh-Doust $^{1}$. Olesya Dulya ${ }^{1,68} \cdot$ Peter Johnston ${ }^{69} \cdot$ Petr Kohout $^{11} \cdot$ Qian Chen $^{61} \cdot$ Qing Tian $^{24} \cdot$ Rajasree Nandi $^{70}$. Rasekh Amiri ${ }^{1}$ Rekhani Hansika Perera ${ }^{24}$ - Renata dos Santos Chikowski ${ }^{32}$ • Renato L. Mendes-Alvarenga ${ }^{32}$. Roberto Garibay-Orijel ${ }^{38} \cdot$ Robin Gielen $^{1} \cdot$ Rungtiwa Phookamsak $^{47} \cdot$ Ruvishika S. Jayawardena $^{24}$. Saleh Rahimlou ${ }^{1}$. Samantha C. Karunarathna ${ }^{47}$. Saowaluck Tibpromma ${ }^{47}$. Shawn P. Brown ${ }^{71}$. Siim-Kaarel Sepp ${ }^{1}$. Sunil Mundra ${ }^{7,72}$. Zhu-Hua Luo ${ }^{73} \cdot$ Tanay Bose $^{74} \cdot$ Tanel Vahter $^{1} \cdot$ Tarquin Netherway $^{67} \cdot$ Teng Yang $^{75} \cdot$ Tom May $^{76} \cdot$ Torda Varga $^{60}$. Wei $\mathrm{Li}^{77}$. Victor Rafael Matos Coimbra ${ }^{32}$. Virton Rodrigo Targino de Oliveira ${ }^{32}$. Vitor Xavier de Lima ${ }^{32}$. Vladimir S. Mikryukov ${ }^{1}$. Yongzhong $\mathrm{Lu}^{78}$. Yosuke Matsuda ${ }^{79}$. Yumiko Miyamoto ${ }^{80}$. Urmas Köljalg ${ }^{1,2}$. Leho Tedersoo ${ }^{1,2}$

Published online: 15 February 2021

(c) MUSHROOM RESEARCH FOUNDATION 2021

\section{Correction to: Fungal Diversity (2020) 105:116}

\author{
https://doi.org/10.1007/s13225-020-00466-2
}

There were errors in the name of author László G. Nagy and in affiliation no. 31 in the original publication. The original article has been corrected.

The original article can be found online at https://doi.org/10.1007/ s13225-020-00466-2.

Sergei Põlme

sergei.polme@gmail.com

Extended author information available on the last page of the article 


\section{Authors and Affiliations}

Sergei Põlme ${ }^{1,2} \mathbb{1} \cdot$ Kessy Abarenkov ${ }^{2} \cdot$ R. Henrik Nilsson ${ }^{3,4} \cdot$ Björn D. Lindahl ${ }^{5} \cdot$ Karina Engelbrecht Clemmensen ${ }^{6}$. Havard Kauserud $^{7} \cdot$ Nhu Nguyen $^{8} \cdot$ Rasmus Kjøller $^{9} \cdot$ Scott T. Bates $^{10} \cdot$ Petr Baldrian $^{11} \cdot$ Tobias Guldberg Frøslev $^{12}$. Kristjan Adojaan ${ }^{1}$ - Alfredo Vizzini ${ }^{13}$. Ave Suija ${ }^{1}$. Donald Pfister ${ }^{14} \cdot$ Hans-Otto Baral $^{15} \cdot$ Helle Järv $^{16}$. Hugo Madrid ${ }^{17,18}$. Jenni Nordén ${ }^{19}$. Jian-Kui Liu ${ }^{20}$. Julia Pawlowska ${ }^{21} \cdot$ Kadri Põldmaa $^{1} \cdot$ Kadri Pärtel $^{1}$. Kadri Runnel ${ }^{1} \cdot$ Karen Hansen $^{22} \cdot$ Karl-Henrik Larsson $^{3,23} \cdot$ Kevin David Hyde $^{24} \cdot$ Marcelo Sandoval-Denis $^{25}$. Matthew E. Smith ${ }^{26} \cdot$ Merje Toome-Heller $^{27}$. Nalin N. Wijayawardene ${ }^{28} \cdot$ Nelson Menolli Jr. $^{29,30}$. Nicole K. Reynolds ${ }^{26} \cdot$ Rein Drenkhan $^{31}$ - Sajeewa S. N. Maharachchikumbura ${ }^{20}$ - Tatiana B. Gibertoni ${ }^{32}$. Thomas Læssøe ${ }^{33}$. William Davis ${ }^{34}$ - Yuri Tokarev ${ }^{35}$. Adriana Corrales ${ }^{36}$. Adriene Mayra Soares ${ }^{37}$. Ahto Agan ${ }^{1}$. Alexandre Reis Machado ${ }^{32}$. Andrés Argüelles-Moyao ${ }^{38}$. Andrew Detheridge ${ }^{39} \cdot$ Angelina de Meiras-Ottoni $^{32}$. Annemieke Verbeken ${ }^{40}$. Arun Kumar Dutta ${ }^{41}$ - Bao-Kai Cui ${ }^{42}$. C. K. Pradeep ${ }^{43}$. César Marín ${ }^{44,45}$. Daniel Stanton ${ }^{46}$. Daniyal Gohar ${ }^{1}$. Dhanushka N. Wanasinghe ${ }^{47}$. Eveli Otsing ${ }^{1}$ · Farzad Aslani ${ }^{1}$. Gareth W. Griffith ${ }^{39}$.

Thorsten H. Lumbsch ${ }^{48}$. Hans-Peter Grossart ${ }^{49,50}$. Hossein Masigol ${ }^{51}$ • Ina Timling ${ }^{52}$ • Inga Hiiesalu ${ }^{1}$. Jane Oja ${ }^{1}$. John Y. Kupagme ${ }^{1}$. József Geml ${ }^{53}$. Julieta Alvarez-Manjarrez ${ }^{38} \cdot$ Kai Ilves $^{1}$ - Kaire Loit ${ }^{54} \cdot$ Kalev Adamson $^{31}$. Kazuhide Nara ${ }^{55} \cdot$ Kati Küngas $^{1} \cdot$ Keilor Rojas-Jimenez ${ }^{56} \cdot$ Krišs Bitenieks $^{57}$ • László Irinyi ${ }^{58,59}$ • László G. Nagy ${ }^{60}$. Liina Soonvald $^{54} \cdot$ Li-Wei Zhou $^{61} \cdot$ Lysett Wagner $^{62} \cdot$ M. Catherine Aime ${ }^{63} \cdot$ Maarja Öpik $^{1} \cdot$ María Isabel Mujica $^{64}$. Martin Metsoja ${ }^{1} \cdot$ Martin Ryberg $^{65} \cdot$ Martti Vasar $^{1} \cdot$ Masao Murata $^{55} \cdot$ Matthew P. Nelsen $^{48} \cdot$ Michelle Cleary $^{66}$. Milan C. Samarakoon ${ }^{24} \cdot$ Mingkwan Doilom $^{47} \cdot$ Mohammad Bahram $^{1,67} \cdot$ Niloufar Hagh-Doust $^{1}$. Olesya Dulya ${ }^{1,68} \cdot$ Peter Johnston $^{69} \cdot$ Petr Kohout $^{11} \cdot$ Qian Chen $^{61} \cdot$ Qing Tian $^{24} \cdot$ Rajasree Nandi $^{70}$. Rasekh Amiri ${ }^{1} \cdot$ Rekhani Hansika Perera ${ }^{24}$ - Renata dos Santos Chikowski ${ }^{32}$ - Renato L. Mendes-Alvarenga ${ }^{32}$. Roberto Garibay-Orijel ${ }^{38} \cdot$ Robin Gielen $^{1} \cdot$ Rungtiwa Phookamsak $^{47} \cdot$ Ruvishika S. Jayawardena $^{24}$. Saleh Rahimlou ${ }^{1}$. Samantha C. Karunarathna ${ }^{47}$. Saowaluck Tibpromma ${ }^{47}$. Shawn P. Brown ${ }^{71}$. Siim-Kaarel Sepp ${ }^{1}$. Sunil Mundra,72 . Zhu-Hua Luo ${ }^{73}$. Tanay Bose ${ }^{74} \cdot$ Tanel Vahter $^{1} \cdot$ Tarquin Netherway $^{67} \cdot$ Teng Yang $^{75} \cdot$ Tom May $^{76} \cdot$ Torda Varga $^{60}$. Wei Li $^{77}$. Victor Rafael Matos Coimbra ${ }^{32}$. Virton Rodrigo Targino de Oliveira ${ }^{32}$. Vitor Xavier de Lima ${ }^{32}$. Vladimir S. Mikryukov ${ }^{1}$. Yongzhong Lu $^{78}$. Yosuke Matsuda ${ }^{79}$. Yumiko Miyamoto ${ }^{80}$. Urmas Kõljalg ${ }^{1,2}$. Leho Tedersoo ${ }^{1,2}$

1 Institute of Ecology and Earth Sciences, University of Tartu, 14A Ravila, 50411 Tartu, Estonia

2 Natural History Museum, University of Tartu, 46 Vanemuise Str, 51003 Tartu, Estonia

3 Gothenburg Global Biodiversity Centre, Box 461, 40530 Gothenburg, Sweden

4 Department of Biological and Environmental Sciences, University of Gothenburg, Gothenburg, Sweden

5 Department of Soil and Environment, Swedish University of Agricultural Sciences, Box 7014, 75007 Uppsala, Sweden

6 Department of Forest Mycology and Plant Pathology, Swedish University of Agricultural Sciences, Box 7026, 75007 Uppsala, Sweden

7 Section for Genetics and Evolutionary Biology (EvoGene), Department of Biosciences, University of Oslo, P.O. Box 1066, Blindern, NO-0316 Oslo, Norway

8 University of Hawai'i at Mānoa, 3190 Maile Way, St. John 102, Honolulu, HI, USA

9 Department of Biology, University of Copenhagen, Universitetsparken 15, 2100 Copenhagen Ø, Denmark

10 Purdue University Northwest, Westville, IN 46391, USA

11 Institute of Microbiology, Czech Academy of Sciences, Videnska 1083, 14220 Praha 4, Czech Republic
12 GLOBE Institute, University of Copenhagen, Øster Farimagsgade 5, KH7.2.32, 1353 Copenhagen, Denmark

13 Dipartimento di Scienze della Vita e Biologia dei Sistemi, Università di Torino, Viale Mattioli 25, 10125 Torino, Italy

14 Department of Organismic and Evolutionary Biology and Farlow Library and Herbarium, Harvard University, 22 Divinity Ave, Cambridge, MA 02138, USA

15 Blaihofstr. 42, 72074 Tübingen, Germany

16 SYNLAB Estonia, Veerenni 53a, Tallinn 11313, Estonia

17 Escuela de Tecnología Médica, Universidad Santo Tomás, Los Carreras 753, Osorno, Chile

18 Centro de Genómica y Bioinformática, Universidad Mayor, Camino La Pirámide 5750, Huechuraba, Santiago, Chile

19 Norwegian Institute for Nature Research (NINA), Sognsveien 68, 0855 Oslo, Norway

20 School of Life Science and Technology, University of Electronic Science and Technology of China, Chengdu 611731, People's Republic of China

21 Evolutionary Biology, Faculty of Biology, Biological and Chemical Research Centre, University of Warsaw, ul. Zwirki i Wigury 101, 02-089 Warsaw, Poland

22 Department of Botany, Swedish Museum of Natural History, P.O. Box 50007, 10405 Stockholm, Sweden

23 Gothenburg Global Biodiversity Centre, P.O. Box 461, 40530 Gothenburg, Sweden 
24 Center of Excellence in Fungal Research, Mae Fah Luang University, Chiang Rai 57100, Thailand

25 Westerdijk Fungal Biodiversity Institute, Utrecht, The Netherlands

26 Department of Plant Pathology, University of Florida, Gainesville, FL, USA

27 Plant Health and Environment Laboratory, Ministry for Primary Industries, Auckland 1140, New Zealand

28 Center for Yunnan Plateau Biological Resources Protection and Utilisation, Qujing Normal University, Qujing 655011, Yunnan, China

29 Instituto Federal de Educação, Ciência e Tecnologia de São Paulo (IFSP), Câmpus São Paulo, Rua Pedro Vicente 625, Canindé, São Paulo, SP 01109-010, Brazil

30 Instituto de Botânica, Núcleo de Pesquisa em Micologia, Av. Miguél Stefano 3687, Água Funda, São Paulo, SP 04301-012, Brazil

31 Institute of Forestry and Rural Engineering, Estonian University of Life Sciences, Fr. R. Kreutzwaldi 5, 51006 Tartu, Estonia

32 Departamento de Micologia, Universidade Federal de Pernambuco, Avenida da Engenharia, S/N - Cidade Universitária, 50740-600 Recife, PE, Brazil

33 Department of Biology \& Globe Institute, University of Copenhagen, Universitetsparken 15, 2100 Copenhagen $\varnothing$, Denmark

34 ARS Research Participation Program, Oak Ridge Institute for Science and Education, Oak Ridge, TN 37830, USA

35 All-Russian Institute of Plant Protection, Podbelskogo 3 Pushkin, St Petersburg 196608, Russia

36 Department of Biology, Universidad del Rosario, Carrera 24 \# 63C-69, Bogota, DC 111221, Colombia

37 Ciências Biológicas, Universidade Federal Rural da Amazônia, Tomé-Açu, Rodovia PA-451, Km 03, 68.680-000, Bairro Açaizal 68.680-000, Brazil

38 Instituto de Biología, Universidad Nacional Autónoma de México, Mexico City, Mexico

39 Institute of Biological, Environmental and Rural Sciences, Aberystwyth University, Wales, UK

40 Research Group Mycology, Department of Biology, Ghent University, Ledeganckstraat 35, 9000 Ghent, Belgium

41 Department of Botany, West Bengal State University, Barasat, North-24-Parganas, West Bengal 700126, India

42 Institute of Microbiology, School of Ecology and Nature Conservation, Beijing Forestry University, Beijing 100083, China

43 Jawaharlal Nehru Tropical Botanic Garden \& Research Institute (JNTBGRI), Palode, Thiruvananthapuram, Kerala 695562, India

44 Institute of Agri-food, Animal and Environmental Sciences (ICA3), Universidad de O'Higgins, 3070000 San Fernando, Chile

45 Center of Applied Ecology and Sustainability (CAPES), Pontificia Universidad Católica de Chile, 8320000 Santiago, Chile
46 Department of Ecology, Evolution and Behavior, University of Minnesota-Twin Cities, Saint Paul, MN 55108, USA

47 CAS Key Laboratory for Plant Diversity and Biogeography of East Asia, Kunming Institute of Botany, Chinese Academy of Sciences, Kunming 650201, Yunnan, China

48 Science and Education and The Grainger Bioinformatics Center, The Field Museum, 1400 S. Lake Shore Dr., Chicago, IL 60605, USA

49 Department of Experimental Limnology, Leibniz Institute of Freshwater Ecology and Inland Fisheries, Alte Fischerhuette 2, 16775 Stechlin, Germany

50 Institute of Biochemistry and Biology, Potsdam University, Maulbeerallee 2, 14469 Potsdam, Germany

51 Department of Plant Protection, University of Guilan, Rasht, Iran

52 Institute of Arctic Biology, University of Alaska, Fairbanks, 311 Irving I Building, 2140 Koyukuk Drive, PO Box 757000, Fairbanks, AK 99775-7000, USA

53 MTA-EKE Lendület Environmental Microbiome Research Group, Eszterházy Károly University, Eger, Hungary

54 Institute of Agricultural and Environmental Sciences, Estonian University of Life Sciences, Tartu, Estonia

55 Department of Natural Environmental Studies, University of Tokyo, 5-1-5 Kashiwanoha, Kashiwa 277-0882, Chiba, Japan

56 Escuela de Biologia, Universidad de Costa Rica, 11501 San Jose, Costa Rica

57 Genetic Resource Centre, Latvian State Forest Research Institute "Silava", 111 Rigas str, Salaspils LV-2169, Latvia

58 Sydney Medical School and Westmead Clinical School and Westmead Institute for Medical Research, University of Sydney, Sydney, NSW, Australia

59 Westmead Hospital, Sydney, NSW, Australia

60 Biological Research Center Szeged, 6726 Szeged, Hungary

61 State Key Laboratory of Mycology, Institute of Microbiology, Chinese Academy of Sciences, Beijing 100101, China

62 National Reference Center for Invasive Fungal Infections (NRZMyk), Leibniz Institute forNatural Product Research and Infection Biology - Hans Knöll Institute (HKI), Jena, Germany

63 Department of Botany and Plant Pathology, Purdue University, West Lafayette, IN 47907, USA

64 Departamento de Ecología, Pontificia Universidad Católica de Chile, Alameda 340, Santiago, Chile

65 Department of Organismal Biology, Uppsala University, Uppsala, Sweden

66 Southern Swedish Forest Research Centre, Swedish University of Agricultural Sciences (SLU), Alnarp, Sweden

67 Department of Ecology, Swedish University of Agricultural Sciences, Uppsala, Sweden

68 Institute of Plant and Animal Ecology, Ural Branch, Russian Academy of Sciences, 8th MarchStreet 202/3, Ekaterinburg, Russia 620144 
69 Manaaki Whenua - Landcare Research, Private Bag 92170, Auckland 1142, New Zealand

70 Institute of Forestry and Environmental Sciences, University of Chittagong, Chittagong, Bangladesh

71 Department of Biological Sciences, University of Memphis, Memphis, TN, USA

72 Department of Biology, College of Science, United Arab Emirates University, Al-Ain, Abu-Dhabi, UAE

73 Key Laboratory of Marine Biogenetic Resources, Third Institute of Oceanography, Ministry of Natural Resources, 184 Daxue Road, Xiamen 361005, China

74 Department of Biochemistry, Genetics and Microbiology, Forestry Agricultural Biotechnology Institute (FABI), University of Pretoria, Pretoria, South Africa
75 State Key Laboratory of Soil and Sustainable Agriculture, Institute of Soil Science, Chinese Academy of Sciences, East Beijing Road 71, Nanjing 210008, China

76 Royal Botanic Gardens Victoria, Melbourne, VIC 3004, Australia

77 College of Marine Life Sciences, Ocean University of China, Qingdao 266003, China

78 School of Food and Pharmaceutical Engineering, Guizhou Institute of Technology, Guiyang 550003, China

79 Graduate School of Bioresources, Mie University, Mie, Japan

80 Arctic Research Center, Hokkaido University, Sapporo, Japan 